\title{
Magnetic test of chiral dynamics in QCD
}

\author{
Yu.A. Simonov \\ Institute of Theoretical and Experimental Physics, \\ 117118, Moscow, B. Cheremushkinskaya 25, Russia \\ E-mail: simonov@itep.ru
}

ABSTRaCt: Strong magnetic fields in the range $e B \gg m_{\pi}^{2}$ effectively probe internal quark structure of chiral mesons and test basic parameters of the chiral theory, such as $\langle\bar{q} q\rangle, f_{\pi}$. We argue on general grounds that $\langle\bar{q} q\rangle$ should grow linearly with $e B$ when charged quark degrees of freedom come into play. To make explicit estimates we extend the previously formulated chiral theory, including quark degrees of freedom, to the case of strong magnetic fields and show that the quark condensate $|\langle\bar{q} q\rangle|_{u, d}$ grows quadratically with $e B$ for $e B<$ $0.2 \mathrm{GeV}^{2}$ and linearly for higher field values. These results agree quantitatively with recent lattice data and differ from $\chi P T$ predictions.

Keywords: Chiral Lagrangians, QCD

ARXIV EPRINT: 1212.3118 


\section{Contents}

1 Introduction 1

2 Effective chiral Lagrangian and the quark condensate 3

3 The MF dependence of quark condensates $\quad 6$

4 Discussion and conclusions $\quad 8$

\section{Introduction}

Strong magnetic fields (MF) are expected to be present in the early universe [1-3], in neutron stars (magnetars) [4-6], and also in non-central heavy-ion collisions [7, 8]. It is very interesting how MF can modify strong interactions, deduced from QCD. In particular, it was recently shown [9], how MF influence the asymptotic freedom behavior of the QCD strong coupling constant and the gluon exchange interaction. The interplay of confinement and MF in neutral mesons was studied in $[10,11]$, showing a dramatic change of masses with growing MF in agreement with recent lattice data [12].

Of a particular interest is the influence of MF on the chiral symmetry breaking (CSB) dynamics, and in a more general setting, on the symmetry breaking pattern in field theory. It was shown in [13], that the GMOR relations in MF hold true for neutral chiral mesons, while they are violated for the charged ones for $e B>0.2 \mathrm{GeV}^{2}$.

It was emphasized in [14-19], in the framework of the NJL model, that MF has the tendency to strengthen the chiral condensate, the phenomenon called there the magnetic catalysis, see [20] for a review. In [21] in the framework of the same model a possible new effect of the magnetic inhibition was predicted.

The behavior of chiral condensate in MF was studied in the chiral perturbation theory $(\chi P T)$ [22-28], in Nambu-Jona-Lasinio (NJL) [29] and in other models [30-32]. On the lattice the corresponding analysis was done for the quenched QCD in $[33,34]$, in $n_{f}=$ $2 Q C D$ in [35-37] and in $n_{f}=4 \mathrm{SU}(2)$ theory in [38, 39]. In all cases both in the models and on the lattice the chiral condensate grows with MF, however in different ways.

Recently a comprehensive analysis of this problem on the lattice with physical quark masses was performed in [40] and has shown a quadratic growth of condensate up to $e B \sim 0.2 \mathrm{GeV}^{2}$ and approximately linear behavior above this value.

This behavior contradicts the $(\chi P T)$ results, see e.g. [22, 28], where a linear growth was found with the slope almost twice as small as in [40], and also contradicts the NJL quadratic growth at large $e B$ [29].

As it was mentioned in $[26,27]$, the reasonable region of $\chi P T$ application is for $e B \lesssim$ $m_{\pi}^{2}$, where the quark condensate grows quadratically, in a qualitative agreement with lattice data, however the linear behavior up to $1 \mathrm{GeV}^{2}$ is outside of the $\chi P T$ reliable region. 
Indeed, the physical degrees of freedom in $\chi P T$ are associated with the strucrureless Nambu-Goldstone mesons, and not with quarks and antiquarks, the role of the latter becomes more important with growing $\mathrm{MF}$, and should be decisive for $e B \gtrsim \sigma$, where $\sigma=0.18 \mathrm{GeV}^{2}$ is the string tension. As it was shown in $[10,11]$ quark and antiquark are strongly attracted to each other in the plain perpendicular to MF, as it follows from the linear growth of the corresponding probability: $\left|\psi_{q \bar{q}}(0)\right|^{2} \sim e B$.

This phenomenon was called in [41] the magnetic focusing, and is the origin of the strong enhancement of the hyperfine interaction in MF [42]. Moreover, magnetic focusing also can produce a linear amplification with MF of atomic, nuclear and hadronic reaction yields, as shown in [43].

It seems reasonable, that some quantities in the chiral theory, like the chiral condensate, are proportional to $\left|\psi_{q \bar{q}}(0)\right|^{2}$, and therefore should grow linearly with $e B$. Indeed, one can write the quark condensate (in the Euclidean space-time)

$$
\begin{aligned}
|\langle\bar{q} q\rangle|=\left|\left\langle S_{q}(x, x)\right\rangle\right|=\left|\left\langle\left(\frac{1}{m+\hat{D}}\right)_{x x}\right\rangle\right| & =\left|\left\langle\left(\frac{1}{m+\hat{D}}\right)_{x y}\left(\frac{1}{m-\hat{D}}\right)_{y x}(m-\hat{D})_{x}\right\rangle\right| \\
& =\left|\left\langle S_{q}(x, y) S_{\bar{q}}(y, x)(m-\hat{D})_{x}\right\rangle\right|
\end{aligned}
$$

and one can visualize the probability amplitudes of the $q \bar{q}$ emission at the point $x$ and absorption at the point $y$, combining into the factor $\left|\psi_{q \bar{q}}(0)\right|^{2}$. This would bring us the linear behavior

$$
|\langle\bar{q} q\rangle| \sim\left|\psi_{q \bar{q}}(0)\right|^{2} \sim e B,
$$

which is not connected to any chiral degrees of freedom. Therefore one can expect, that in any model, which takes into account the general structure (1.1) of the quark condensate, one would end up with the linear growth (1.2), and the main emphasis should be on the exact quantitative form, i.e. on the coefficient in front of $e B$ in (1.2). Here the recent accurate lattice analysis in [40] gives a good check of analytic results, which will be used below.

It is the purpose of the present paper to study the quark condensate in MF starting from the basic QCD equations, derived earlier without MF in [44-48]. It was shown there, that GMOR relations and expressions for $\langle\bar{q} q\rangle$ and $f_{\pi}$ can be derived from the basic QCD quantities: string tension, $\alpha_{s}$ and current quark masses in good agreement with experiment and lattice data.

Recently in [49] these results were extended to account for growing current quark masses $m_{q}$, and in particular the dependence of $\langle\bar{q} q\rangle$ on $m_{q}$ was established to be in agreement with lattice data [50-54].

In the present paper we follow the same line of the formalism of [44-48], but now adding MF, we find the behavior of $\langle\bar{q} q\rangle$ for $u$ and $d$ quarks and their average with growing MF.

As a result we observe in the resulting dependence of the average $\langle\bar{q} q\rangle$ and $\langle\bar{u} u\rangle,\langle\bar{d} d\rangle$, the same features and good quantitative agreement with the lattice data obtained in [40]. The physical reason for this dependence of $\langle\bar{q} q\rangle$ on $e B$ is clarified below in the paper.

The paper is organized as follows. In the next section a general derivation of GMOR relation and expressions for $\langle\bar{q} q\rangle$ and $f_{\pi}$ are given, in section 3 the MF dependence of basic terms is established, in section 4 results are discussed and prospectives are given. 


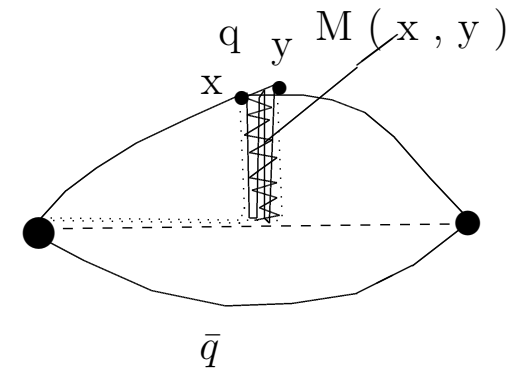

Figure 1. The flux-tube operator $M(x, y)$ in the Wilson loop.

\section{Effective chiral Lagrangian and the quark condensate}

We start with the situation without MF. In this case in $[47,48]$ the effective Lagrangian for Nambu-Goldstone (NG) mesons with $q \bar{q}$ degrees of freedom was obtained in the form

$$
L_{\mathrm{ECL}}=N_{c} \operatorname{tr} \log \left[\left(\hat{\partial}+m_{f}\right) \hat{1}+M \hat{U}\right]
$$

where

$$
\hat{U}=\exp \left(i \gamma_{5} \hat{\phi}\right)
$$

and $M(x)$ in the local limit is a quark confining interaction, $M(x) \sim \sigma|\mathbf{x}|$ for $|\mathbf{x}| \rightarrow \infty$, see figure 1 , while $\hat{\phi}=\phi_{a} t^{a}, \phi_{a}(x)$ is the octet of NG mesons.

It is important, that $M(x)$, appearing at the vertices of the Green's function in figure 1, are finite,

$$
M(0) \cong \frac{2 \sigma \lambda}{\sqrt{\pi}}\left(1+O\left(\sigma \lambda^{2}\right)\right.
$$

where $\lambda$ is the vacuum correlation length, $\lambda \cong(0.1 \div 0.15)$ fm $[55-58]$.

Expanding (2.1) to the quadratic in $\phi$ terms, one obtains the GMOR relation, e.g. for the pion

$$
m_{\pi}^{2} f_{\pi}^{2}=\frac{\bar{m}_{u}+\bar{m}_{d}}{2}|\langle\bar{\psi} \psi\rangle|, \quad \bar{m}=\frac{m_{u}+m_{d}}{2},
$$

where the quark condensate $\langle\bar{\psi} \psi\rangle$ is

$$
\langle\bar{\psi} \psi\rangle_{M}=i\left\langle\psi \psi^{+}\right\rangle_{E}=-N_{c} \operatorname{tr} \Lambda, \quad \Lambda=(\hat{\partial}+\bar{m}+M)^{-1} .
$$

It was also found in $[45,46]$, that $\operatorname{tr} \Lambda$ can be expressed via the $q \bar{q}$ Green's function $G^{(0)}(k)$

$$
\begin{aligned}
\operatorname{tr} \Lambda & =\left\langle\operatorname{tr} \frac{1}{M+m_{q}+\hat{\partial}}\right\rangle=\left\langle\operatorname{tr}\left[\frac{1}{M+m_{q}+\hat{\partial}}\left(M+m_{q}-\hat{\partial}\right) \frac{1}{\left(M+m_{q}-\hat{\partial}\right)}\right]\right\rangle \\
& =\left(M(0)+m_{q}\right)\left\langle\operatorname{tr}\left[\gamma_{5} \frac{1}{M+m_{q}+\hat{\partial}} \gamma_{5} \frac{1}{M+m_{q}+\hat{\partial}}\right]\right\rangle,
\end{aligned}
$$

and

$$
\operatorname{tr} \Lambda=-(M(0)+m) G^{(0)}(k=0)
$$


where the spectral decomposition is $[45,46]$

$$
G^{(0)}(k)=-\sum_{n=0} \frac{c_{n}^{2}}{k^{2}+m_{n}^{2}}
$$

and $c_{n}=\sqrt{\frac{m_{n}}{2}} \psi_{n}(0)$. Finally, the quark condensate for $i=u, d, s$ quarks is

$$
-\langle\bar{q} q\rangle_{i}=N_{c}\left(M(0)+m_{i}\right) \sum_{n=0}^{N} \frac{\psi_{n}^{2}(0)}{m_{n}} e^{-m_{n} \lambda}, \quad i=u, d, s
$$

eq. (2.8) expresses the quark condensate in terms of $M(0)$ and the reference spectrum in the PS channel, i.e. the spectrum, where chiral symmetry is not taken into account, but only confinement term $M(x)$ is present, the corresponding masses $m_{n}$, calculated in $[45,46]$ are $m_{0}=0.4 \mathrm{GeV}, m_{1}=1.35 \mathrm{GeV}, m_{2}=1.85 \mathrm{GeV}$. Taking $\lambda=0.1 \mathrm{fm}$ and $M(0)=0.15 \mathrm{GeV}$, one obtains (cf appendix of the second paper in $[45,46]$ )

$$
-\frac{\langle\bar{q} q\rangle}{n_{f}}=(217 \mathrm{MeV})^{3} \frac{M(0)+\bar{m}}{(150 \mathrm{MeV})}, \quad f_{\pi}=96 \mathrm{MeV} \frac{M(0)+\bar{m}}{(150 \mathrm{MeV})} .
$$

One can see, that our values are in the correct ballpark, close to average lattice and phenomenological values.

Now we turn to the case of a nonzero MF. In this case one should replace $\partial_{\mu} \rightarrow D_{\mu} \equiv$ $\partial_{\mu}-i e_{f} A_{\mu}^{(e)}$ and therefore $\Lambda_{+/-}=\frac{1}{m+M+\hat{D}_{+/-}}$, where $\hat{D}_{+/-}=\hat{\partial}_{\mp} i e_{f} \hat{A}^{(e)}$. Also one should introduce $\bar{\Lambda}_{+/-}=\frac{1}{m+M-\hat{D}_{+/-}}$, so that the quark condensate now contains contribution of both quarks and antiquarks,

$$
\begin{aligned}
\langle\bar{\psi} \psi\rangle_{M, i} & =-N_{c}\left(M(0)+m_{i}\right) \operatorname{tr} \Lambda_{+} \bar{\Lambda}_{-} \\
& =-N_{c}\left(M(0)+m_{i}\right) \operatorname{tr}\left(\Lambda_{+} \gamma_{5} \Lambda_{-} \gamma_{5}\right)=-(M(0)+m) G^{(B)}(k) .
\end{aligned}
$$

Moreover, MF destroys both spin and isospin quantum numbers of the meson, and therefore one must distinguish in the Green's function of our neutral $q \bar{q}$ system not only $(u \bar{u})$ and $(d \bar{d})$ components separately, but also the $q \bar{q}$ spin projections $(+-)$ and $(-+)$, since they correspond to different mass eigenvalues.

As a result eq. (2.8) in case of MF can be rewritten for each flavor separately,

$$
\left|\langle\bar{q} q\rangle_{i}\right|=N_{c}\left(M(0)+m_{i}\right) \sum_{n=0}^{\infty}\left(\frac{\frac{1}{2}\left|\psi_{n, i}^{(+-)}(0)\right|^{2}}{m_{n, i}^{(+-)}}+\frac{\frac{1}{2}\left|\psi_{n, i}^{(-+)}(0)\right|^{2}}{m_{n, i}^{(-+)}}\right)
$$

where $i=u, d, s$ and the superscripts $(+-)$ and $(-+)$ refer to the quark and antiquark spin projections on the MF $\mathbf{B}$, and the coefficients $\frac{1}{2}$ are due to $\gamma_{5}$ in the vertices of the Green's function $G^{(0)}(k),\left\langle\gamma_{5}\right| \rightarrow \frac{\langle+-|-\langle-+|}{\sqrt{2}}$.

The main problem reduces to the calculation of the spectrum of eigenvalues and eigenfunctions $\psi_{n}, m_{n}$, which are to be found from the Hamiltonian $H$ containing MF $\mathbf{B}$, and derived from the path integral Hamiltonian [10, 11]

$$
H_{0}=\sum_{i=1}^{2}\left(\frac{\left(\mathbf{p}_{i}-e_{i} \mathbf{A}^{(e)}\right)^{2}}{2 \omega_{i}}+\frac{m_{i}^{2}+\omega_{i}^{2}}{2 \omega_{i}}-\frac{e_{i} \boldsymbol{\sigma}_{i} \mathbf{B}}{2 \omega_{i}}\right)
$$


which can be rewritten in the form $(i=u, d, s)$

$$
H_{i}=\frac{1}{2 \tilde{\omega}}\left(-\frac{d^{2}}{d \boldsymbol{\eta}^{2}}+\left(\frac{e_{i}(\boldsymbol{\eta} \times \mathbf{B})}{2}\right)^{2}\right)+V_{\mathrm{conf}}(\eta)-\sum_{k=1,2} \frac{e_{i} \boldsymbol{\sigma}_{k} \mathbf{B}}{2 \omega_{k}},
$$

where $\tilde{\omega}=\frac{\omega_{1} \omega_{2}}{\omega_{1}+\omega_{2}}$.

The eigenvalues $\tilde{m}_{n, i}$ of $H_{i}$ depend on $\omega_{1}, \omega_{2}$, and the final eigenvalues $m_{n, i}$ entering in (2.11) are obtained as stationary points in variation over $\omega_{1}, \omega_{2}$

$$
\left.\frac{\partial \tilde{m}_{n, i}}{\partial \omega_{1,2}}\right|_{\omega_{1,2}^{(0)}}=0, \quad \text { e.g. } \tilde{m}_{n, i}^{(+-)}\left(\omega_{1}^{(0)}, \omega_{2}^{(0)}\right) \equiv m_{n, i}^{(+-)} .
$$

To avoid purely numerical calculations, one can simplify the hamiltonian (2.13), replacing linear confinement $V_{\text {conf }}(\eta)$ in (2.13) by a suitable quadratic form, with coefficient found from the stationary point condition

$$
V_{\text {conf }}^{(\text {lin })}=\sigma \eta \rightarrow V_{\text {conf }}^{(\text {quadr })}=\frac{\sigma}{2}\left(\eta^{2} \gamma+\frac{1}{\gamma}\right),\left.\quad \frac{\partial \tilde{m}_{n, i}}{\partial \gamma}\right|_{\gamma=\gamma_{0}}=0
$$

To check the accuracy of this replacement, one can compare the lowest mass eigenvalues and $\psi_{n}(0)$ first without MF. In particular, $m_{0}^{(\text {quadr })}=2 \sqrt{3 \sigma}$, while $m_{0}^{\text {lin }}=4 \sqrt{\sigma}\left(\frac{2.338}{3}\right)^{3 / 4}$, and these two figures differ by $4.5 \%$.

For $\psi_{n}(0)$ the corresponding results are

$$
\begin{aligned}
\left|\psi_{n}(0)\right|_{\text {lin }}^{2} & =\frac{0.82 \sigma^{3 / 2}}{4 \pi}=0.065 \sigma^{3 / 2} \\
\left|\psi_{0}(0)\right|_{\text {quadr }}^{2} & =\frac{\sigma^{3 / 2}\left(\frac{c_{0}}{4}\right)^{3 / 4}}{\pi^{3 / 2}}=0.065 \sigma^{3 / 2} \quad\left(c_{0} \equiv 1\right)
\end{aligned}
$$

This coincidence of $\left|\psi_{0}(0)\right|^{2}$ will be of special importance in what follows, since the main effect of MF, as will be seen, is the increase of $\left|\psi_{n}(0)\right|^{2}$ in (2.11) due to MF. It is clear, that with growing $B$ the size of meson is decreasing, and the difference between $V_{\text {conf }}^{(\text {lin }}$ and $V_{\text {conf }}^{\text {(quadr) }}$ will be even more suppressed, since these both interactions vanish simultaneously, $V_{\text {conf }}^{\text {(lin) }}(r \rightarrow 0)=V_{\text {conf }}^{\text {(quadr) }}(r \rightarrow 0)=0$.

For us it will be most important how $\left|\psi_{n}(0)\right|^{2}$ depends on MF, and especially, how MF enters in the expansion of $\left|\psi_{n}(0)\right|^{2}$ in powers of $B$. To this end one can see in the Hamiltonian (2.13), that MF enters via the term $V_{B} \equiv \frac{\boldsymbol{\eta}_{\perp}^{2}}{2 \tilde{\omega}}\left(\frac{e_{i} \mathbf{B}}{2}\right)^{2}$. It is clear, that in the perturbative series expansion the MF enters as $\left(e_{i} B\right)^{2}$

$$
\psi_{n}(0)_{B}=\psi_{n}(0)_{0}+O\left(\left(e_{i} B\right)^{2}\right)+\ldots
$$

As a result of (2.15) one can immediately write the analytic expressions for $\psi_{n}(0)$ and $m_{n}$ of the following form, e.g.

$$
\left|\psi_{n}(0)\right|^{2}=\frac{1}{\pi^{3 / 2} r_{\perp}^{2} r_{3}}, \quad r_{\perp}^{2}=2\left(\left(e_{q} B\right)^{2}+\sigma^{2} c\right)^{-1 / 2}, \quad r_{3}=\left(\frac{\sigma^{2} c}{4}\right)^{-1 / 4}
$$


where $c=\frac{4 \tilde{\omega}}{\gamma \sigma}$, and

$$
m_{n_{\perp}, n_{3}}=\varepsilon_{n_{\perp}, n_{3}}+\frac{m_{1}^{2}+\omega_{1}^{2}-e_{q} \mathbf{B} \boldsymbol{\sigma}_{1}}{2 \omega_{1}}+\frac{m_{2}^{2}+\omega_{2}^{2}+e_{q} \mathbf{B} \boldsymbol{\sigma}_{2}}{2 \omega_{2}}
$$

where

$$
\varepsilon_{n_{\perp}, n_{3}}=\frac{1}{2 \tilde{\omega}}\left[\sqrt{e_{q}^{2} B^{2}+\sigma^{2} c}\left(2 n_{\perp}+1\right)+\sqrt{\sigma^{2} c}\left(n_{3}+\frac{1}{2}\right)\right]+\frac{\gamma \sigma}{2}
$$

Expressions (2.11), (2.18), (2.19), (2.20) contain all information necessary to compute the quark condensate for a varying MF.

\section{The MF dependence of quark condensates}

We are interested in eigenfunctions and eigenvalues of $(+-)$ and $(-+)$ states both for $u$ and $d$ quarks. For the $(+-)$ state, one can see in $(2.20),(2.19)$ that at large $B, m_{0, n_{3}}^{(+-)}$ tends to a constant limit, together with $\omega_{1}=\omega_{2}$. In this case the parameter $c$ in $(2.18)$ and (2.20) can be expressed in general as

$$
c_{+-}=\frac{4 \tilde{\omega}}{\gamma \sigma}=\left(\frac{a}{2}\right)^{4 / 3} 4 / \beta ; \quad \gamma(B)=\beta(B)(\sigma \tilde{\omega})^{-1 / 3}, \quad \omega(B)=a(B) \sqrt{\sigma}
$$

and $\beta(B), a(B)$ are changing in finite limits and one obtains that $c_{+-}(B) \approx 1$, for all $B$, and $m_{+-}$tends to a constant limit for $B \rightarrow \infty$. Hence one can write for lowest levels $n_{3}=0,1,2, \ldots$

$$
\left|\psi_{n_{\perp}=0, n_{3}}^{(+-)}(0)\right|^{2} \cong \frac{\sqrt{\sigma} \sqrt{e_{q}^{2} B^{2}+\sigma^{2}}}{(2 \pi)^{3 / 2}} .
$$

Note, that in the limit $B \rightarrow 0$ this expression for $|\psi(0)|^{2}$ yields equal values for $n_{3}=0,1,2$ as it should be for pure linear confining interaction.

For the $(-+)$ case the situation is different, and at large $B$ the stationary point value $\omega_{0}^{(-+)} \approx \sqrt{2\left|e_{q}\right| B+\frac{\sigma}{4}}$, and the parameter $c_{-+}$is increasing with $B$ :

$$
c_{-+}(B)=\left(1+\frac{8 e_{q} B}{\sigma}\right)^{2 / 3} \text {. }
$$

As a result, the $\left|\psi_{n}^{(-+)}(0)\right|^{2}$ can be written as

$$
\left|\psi_{n_{3}}^{(-+)}(0)\right|^{2}=\left(\sigma^{2} c_{-+}\right)^{3 / 4} \sqrt{1+\left(\frac{e_{q} B}{\sigma}\right)^{2} \frac{1}{c_{-+}}} .
$$

Moreover, $m_{n_{3}}^{(-+)} \approx 2 \sqrt{2\left|e_{q} B\right|+\frac{\sigma}{4}}$, and $\frac{\left(\sigma^{2} c_{-+}\right)^{3 / 4}}{m_{0}^{(-+)}(B)}=\sigma$ at large $B$.

Combining (2.20) and (3.4) we therefore can write

$$
\left|\langle\bar{q} q\rangle_{i}(B)\right|=\left|\langle\bar{q} q\rangle_{i}(0)\right| \frac{1}{2}\left\{\sqrt{1+\left(\frac{e_{q} B}{\sigma}\right)^{2}}+\sqrt{1+\left(\frac{e_{q} B}{\sigma}\right)^{2} \frac{1}{c_{-+}}}\right\}
$$

where $c_{-+}$is given in (3.3). 


\begin{tabular}{|l|l|l|l|l|l|l|}
\hline $\begin{array}{l}e B \\
\left(\mathrm{GeV}^{2}\right)\end{array}$ & 0 & 0.2 & 0.4 & 0.6 & 0.8 & 1 \\
\hline $\begin{array}{l}\Delta \sum_{u} \\
\text { this paper }\end{array}$ & 0 & 0.156 & 0.48 & 0.865 & 1.273 & 1.65 \\
\hline $\begin{array}{l}\Delta \sum_{u}[21] \\
\text { lattice }\end{array}$ & 0 & 0.185 & 0.51 & 0.86 & 1.235 & 1.60 \\
\hline $\begin{array}{l}\Delta \sum_{d} \\
\text { this paper }\end{array}$ & 0 & 0.048 & 0.158 & 0.308 & 0.48 & 0.67 \\
\hline $\begin{array}{l}\Delta \sum_{d}[21] \\
\text { lattice }\end{array}$ & 0 & 0.095 & 0.23 & 0.40 & 0.57 & 0.73 \\
\hline
\end{tabular}

Table 1. Values of $\Delta \sum_{i}(B), i=u, d$ given by (3.5) in comparison with lattice data from [40]

We shall be using notations of [40] for the increment of the quark condensate as a function of MF (denoted $\tau_{i}(B)$ in [14-19])

$$
\Delta \sum_{i}(B)=\frac{\left|\langle\bar{q} q\rangle_{i}(B)\right|-\left|\langle\bar{q} q\rangle_{i}(0)\right|}{\left|\langle\bar{q} q\rangle_{i}(0)\right|} .
$$

The resulting values of $\Delta \sum_{i}(B)$ from (3.5), (3.6) are compared in table 1 with the corresponding lattice calculations in [40].

One can see a reasonable agreement between our theory and lattice data. One can simplify the $B$ dependence of eq. (3.5), writing

$$
\left|\langle\bar{q} q\rangle_{i}(B)\right|=\left|\langle\bar{q} q\rangle_{i}(0)\right| \sqrt{1+\left(\frac{e_{i} B}{M_{i}^{2}}\right)^{2}} .
$$

The form (3.7) also satisfactorily describes data of [36, 37] and [40], taking $M_{i}$ as a fitting parameter. In the case of $[36,37]$ the fitted values of $M_{i}$ are approximately

$$
M_{u}^{2} \approx 0.35 \mathrm{GeV}^{2}, \quad M_{d}^{2} \approx 0.27 \mathrm{GeV}^{2},
$$

i.e. are 2 and 1.5 times larger, than $\sigma$ (the corresponding $c=c_{+-}=c_{-+} \cong 4$ and 4.25 for $u$ and $d$ cases), however the agreement with lattice data is worse. Qualitatively the same situation (with even larger $M_{i}^{2}$ ) takes place in comparison with [33, 34].

As a whole the behavior (3.5), (3.7) correctly reproduces the main qualitative features of the quark condensate as a function of $e B$ : the quadratic behavior proportional to $\left(e_{i} B\right)^{2}$ at small $B$ and linear behavior $\sim\left|e_{i} B\right|$ at large $B$. It differs from the results of others approaches. In particular, $\chi P T[22-25,28]$ predicts linear behavior in $e B$ with the slope much smaller, than in lattice data [40].

As it was mentioned in [36, 37], for $\Delta \sum_{u}$ and $\Delta \sum_{d}$ there is a simple relation

$$
\Delta \sum_{u}\left(\frac{B}{2}\right)=\Delta \sum_{d}(B),
$$

which is satisfied in lattice data, and, of course, is trivially satisfied in our definitions.

Writing for large $B \gg \sigma$, that $\Delta \sum_{i}(B) \cong a_{i} B$, one immediately obtains from (3.9), that $a_{u}=2 a_{d}$. This relation is approximately satisfied in lattice data [36, 37, 40], and in our expression (3.5), (3.7). 


\section{Discussion and conclusions}

We have used our formalism for the chiral dynamics, presented in [44-49], which is derived not from purely symmetry considerations, but from the QCD quark dynamics, where chiral symmetry appears approximately in the small $m_{q}$ limit of the effective QCD Lagrangian. In this way all basic degrees of freedom are connected to the confined quarks, and it is finally the confinement, which dictates the properties of fundamental chiral quantities $\langle\bar{q} q\rangle, f_{\pi}$ etc., and gives them numerical values, expressed via $\sigma$. This is in contrast with standard chiral ideas, where chiral Lagrangian is derived on the basis of the chiral symmetry breaking, however the final results, like GMOR relations are the same.

Actually our reasoning for the calculation of quark condensate is very simple. After one derives scalar confining interaction $M(x)$, which acts on each quark or antiquark, one can write quark condensate $\langle\bar{q} q\rangle=-N_{c} \operatorname{tr} \Lambda$ as

$$
\begin{aligned}
\operatorname{tr} \Lambda & =\left\langle\operatorname{tr} \frac{1}{M+m_{q}+\hat{\partial}}\right\rangle=\left\langle\operatorname{tr}\left[\frac{1}{M+m_{q}+\hat{\partial}}\left(M+m_{q}-\hat{\partial}\right) \frac{1}{\left(M+m_{q}-\hat{\partial}\right)}\right]\right\rangle \\
& =\left(M(0)+m_{q}\right)\left\langle\operatorname{tr}\left[\gamma_{5} \frac{1}{M+m_{q}+\hat{\partial}} \gamma_{5} \frac{1}{M+m_{q}+\hat{\partial}}\right]\right\rangle,
\end{aligned}
$$

since the term with $\hat{\partial}$ is odd and vanishes.

The last quantity on the r.h.s. of (4.1) is the $q \bar{q}$ Green's function, proportional to $\psi_{n}^{2}(0)$ for each $n$ state. The only difference in presence of MF is the replacement $\hat{\partial} \rightarrow\left(\hat{\partial}-i e \hat{A}^{(e)}\right)$, which immediately gives the proportionality $\langle\bar{q} q\rangle \sim \psi^{2}(r=0 ; e B)$. This latter quantity is linearly rising with $|e B|$, since MF is "focusing" the quark-antiquark system at small distances. Note the similarity of (4.1) and (1.1).

This phenomenon of the "magnetic focusing" is of a general character and in the case of the chiral condensate it actually explains dynamically its growth with $e B$, which was named before in [14-20] "magnetic catalysis". Recently the effect of "magnetic focusing" in the hyperfine interaction in hydrogen was studied in [41], and in the case of molecular, nuclear and hadronic processes in [43].

External magnetic field is here crucial for our understanding of chiral dynamics, and using MF one may decide, what is the role of quark dynamics in the chiral phenomena. In this respect the comparison of the behavior of quark condensate (or $\Delta \sum(B)$ ) as a function of $B$ in different models and lattice data is showing the following:

1. Standard chiral theory at large $B \sim \sigma$ gives linear behavior (qualitatively correct) but with the wrong slope.

2. Our approach, eq. (2.11) predicts quadratic behavior at small $B B \lesssim \sigma$ and linear at larger $B$ with slopes different for $u$ and $d$ quarks, both in agreement with existing lattice data.

3. The PNJL model as shown in [40], is also in disagreement with lattice data at larger $e B$. 
Physically, it is clear, that MF, acting on quark charges, discloses the internal quark structure of PS mesons, while the effective chiral Lagrangian, as in [22-25], describes only internal multipionic degrees of freedom. The latter can be important only for $B \ll 1 / r_{0}^{2}$, where $r_{0} \approx 0.6 \mathrm{fm}$ is the pionic radius, i.e. for $B \ll 0.1 \mathrm{GeV}^{2}$, while for large $B$ the standard chiral picture is irrelevant, as it is confirmed by lattice data.

These considerations suggest the idea, that the true chiral dynamics can be derived e.g. from the effective Lagrangian (2.1), and should finally display coexisting quark and chiral symmetric degrees of freedom, demonstrating how the latter are disappearing (suppressed) for growing quark masses. How it happens with the pionic spectra, was explained in [49], demonstrating the unifying spectrum based on chiral and quark degrees of freedom at the same time.

\section{Acknowledgments}

The author is grateful for discussions to N.O.Agasian, M.A.Andreichikov and B.O.Kerbikov.

Open Access. This article is distributed under the terms of the Creative Commons Attribution License (CC-BY 4.0), which permits any use, distribution and reproduction in any medium, provided the original author(s) and source are credited.

\section{References}

[1] T. Vachaspati, Magnetic fields from cosmological phase transitions, Phys. Lett. B 265 (1991) 258 [INSPIRE].

[2] K. Enqvist and P. Olesen, On primordial magnetic fields of electroweak origin, Phys. Lett. B 319 (1993) 178 [hep-ph/9308270] [INSPIRE].

[3] D. Grasso and H.R. Rubinstein, Magnetic fields in the early universe, Phys. Rept. 348 (2001) 163 [astro-ph/0009061] [INSPIRE].

[4] R.C. Duncan and C. Thompson, Formation of very strongly magnetized neutron stars Implications for gamma-ray bursts, Astrophys. J. 392 (1992) L9 [INSPIRE].

[5] J.M. Lattimer and M. Prakash, Neutron star observations: prognosis for equation of state constraints, Phys. Rept. 442 (2007) 109 [astro-ph/0612440] [INSPIRE].

[6] A.K. Harding and D. Lai, Physics of strongly magnetized neutron stars, Rep. Prog. Phys. 69 (2006) 2631 [astro-ph/0606674] [INSPIRE].

[7] D.E. Kharzeev, L.D. McLerran and H.J. Warringa, The effects of topological charge change in heavy ion collisions: 'Event by event $P$ and CP-violation', Nucl. Phys. A 803 (2008) 227 [arXiv:0711.0950] [INSPIRE].

[8] V. Skokov, A.Y. Illarionov and V. Toneev, Estimate of the magnetic field strength in heavy-ion collisions, Int. J. Mod. Phys. A 24 (2009) 5925 [arXiv:0907.1396] [InSPIRE].

[9] M.A. Andreichikov, V.D. Orlovsky and Yu.A. Simonov, Asymptotic freedom in strong magnetic fields, Phys. Rev. Lett. 110 (2013) 162002 [arXiv:1211.6568] [INSPIRE]. 
[10] Yu.A. Simonov, B.O. Kerbikov and M.A. Andreichikov, Quark-Antiquark System in Ultra-Intense Magnetic Field, arXiv:1210.0227 [INSPIRE].

[11] M.A. Andreichikov, B.O. Kerbikov, V.D. Orlovsky and Yu.A. Simonov, Meson spectrum in strong magnetic fields, Phys. Rev. D 87 (2013) 094029 [arXiv: 1304.2533] [INSPIRE].

[12] Y. Hidaka and A. Yamamoto, Charged vector mesons in a strong magnetic field, Phys. Rev. D 87 (2013) 094502 [arXiv: 1209.0007] [InSPIRE].

[13] V. Orlovsky and Y. Simonov, Nambu-Goldstone mesons in strong magnetic field, JHEP 09 (2013) 136 [arXiv:1306.2232] [INSPIRE].

[14] K. Klimenko, Three-dimensional Gross-Neveu model in an external magnetic field, Theor. Math. Phys. 89 (1992) 1161 [INSPIRE].

[15] K. Klimenko, Three-dimensional Gross-Neveu model at nonzero temperature and in an external magnetic field, Theor. Math. Phys. 90 (1992) 1 [INSPIRE].

[16] V. Gusynin, V. Miransky and I. Shovkovy, Catalysis of dynamical flavor symmetry breaking by a magnetic field in $(2+1)$-dimensions, Phys. Rev. Lett. 73 (1994) 3499 [Erratum ibid. 76 (1996) 1005] [hep-ph/9405262] [INSPIRE].

[17] V. Gusynin, V. Miransky and I. Shovkovy, Dynamical flavor symmetry breaking by a magnetic field in (2+1)-dimensions, Phys. Rev. D 52 (1995) 4718 [hep-th/9407168] [INSPIRE].

[18] V. Gusynin, V. Miransky and I. Shovkovy, Dimensional reduction and dynamical chiral symmetry breaking by a magnetic field in (3+1)-dimensions, Phys. Lett. B 349 (1995) 477 [hep-ph/9412257] [INSPIRE].

[19] V. Gusynin, V. Miransky and I. Shovkovy, Dimensional reduction and catalysis of dynamical symmetry breaking by a magnetic field, Nucl. Phys. B 462 (1996) 249 [hep-ph/9509320] [INSPIRE].

[20] I.A. Shovkovy, Magnetic catalysis: a review, Lect. Notes Phys. 871 (2013) 13 [arXiv: 1207.5081] [INSPIRE].

[21] K. Fukushima and Y. Hidaka, Magnetic catalysis vs. magnetic inhibition, Phys. Rev. Lett. 110 (2013) 031601 [arXiv:1209.1319] [INSPIRE].

[22] I. Shushpanov and A.V. Smilga, Quark condensate in a magnetic field, Phys. Lett. B 402 (1997) 351 [hep-ph/9703201] [INSPIRE].

[23] N.O. Agasian and I. Shushpanov, The quark and gluon condensates and low-energy QCD theorems in a magnetic field, Phys. Lett. B 472 (2000) 143 [hep-ph/9911254] [INSPIRE].

[24] N.O. Agasian, Phase structure of the QCD vacuum in a magnetic field at low temperature, Phys. Lett. B 488 (2000) 39 [hep-ph/0005300] [INSPIRE].

[25] N.O. Agasian, Chiral thermodynamics in a magnetic field, Phys. Atom. Nucl. 64 (2001) 554 [hep-ph/0112341] [INSPIRE].

[26] J.O. Andersen, Chiral perturbation theory in a magnetic background - Finite-temperature effects, JHEP 10 (2012) 005 [arXiv: 1205.6978] [INSPIRE].

[27] J.O. Andersen, Thermal pions in a magnetic background, Phys. Rev. D 86 (2012) 025020 [arXiv: 1202.2051] [INSPIRE].

[28] T.D. Cohen, D.A. McGrady and E.S. Werbos, Chiral condensate in a constant electromagnetic field, Phys. Rev. C 76 (2007) 055201. 
[29] R. Gatto and M. Ruggieri, Deconfinement and chiral symmetry restoration in a strong magnetic background, Phys. Rev. D 83 (2011) 034016 [arXiv: 1012.1291] [INSPIRE].

[30] N. Agasian and S. Fedorov, Quark-hadron phase transition in a magnetic field, Phys. Lett. B 663 (2008) 445 [arXiv:0803.3156] [INSPIRE].

[31] E.S. Fraga and A.J. Mizher, Can a strong magnetic background modify the nature of the chiral transition in QCD?, Nucl. Phys. A 820 (2009) 103C [arXiv:0810.3693] [INSPIRE].

[32] E.S. Fraga and L.F. Palhares, Deconfinement in the presence of a strong magnetic background: an exercise within the MIT bag model, Phys. Rev. D 86 (2012) 016008 [arXiv:1201.5881] [INSPIRE].

[33] P. Buividovich, M. Chernodub, E. Luschevskaya and M. Polikarpov, Numerical study of chiral symmetry breaking in non-abelian gauge theory with background magnetic field, Phys. Lett. B 682 (2010) 484 [arXiv:0812.1740] [INSPIRE].

[34] V.Braguta et al., The chiral magnetic effect and chiral symmetry breaking in $\mathrm{SU}(3)$ quenched lattice gauge theory, PoS (LATTICE 2010) 190 [arXiv:1011.3795] [INSPIRE].

[35] M. D'Elia, S. Mukherjee and F. Sanfilippo, QCD phase transition in a strong magnetic background, Phys. Rev. D 82 (2010) 051501 [arXiv: 1005.5365] [INSPIRE].

[36] M. D'Elia and F. Negro, Chiral properties of strong interactions in a magnetic background, Phys. Rev. D 83 (2011) 114028 [arXiv:1103.2080] [INSPIRE].

[37] M. D'Elia, Lattice QCD simulations in external background fields, Lect. Notes Phys. 871 (2013) 181 [arXiv:1209. 0374] [INSPIRE].

[38] E.-M. Ilgenfritz, M. Kalinowski, M. Muller-Preussker, B. Petersson and A. Schreiber, Two-color QCD with staggered fermions at finite temperature under the influence of a magnetic field, Phys. Rev. D 85 (2012) 114504 [arXiv:1203.3360] [INSPIRE].

[39] E.-M. Ilgenfritz, M. Kalinowski, M. Muller-Preussker, B. Petersson and A. Schreiber, Two-color QCD with staggered fermions at finite temperature under the influence of a magnetic field, Phys. Rev. D 85 (2012) 114504 [arXiv:1203.3360] [INSPIRE].

[40] G.S. Bali et al., QCD quark condensate in external magnetic fields, Phys. Rev. D 86 (2012) 071502 [arXiv: 1206.4205] [INSPIRE].

[41] M.A. Andreichikov, B.O. Kerbikov and Yu.A. Simonov, Magnetic field focusing of hyperfine interaction in hydrogen, arXiv:1304.2516 [INSPIRE].

[42] Yu.A. Simonov, Spin interactions in mesons in strong magnetic field, Phys. Rev. D 88 (2013) 053004 [arXiv: 1304.0365] [InSPIRE].

[43] Yu.A. Simonov, Magnetic focusing in atomic, nuclear and hadronic processes, arXiv: 1308.5553 [INSPIRE].

[44] Yu.A. Simonov, Theory of light quarks in the confining vacuum, Phys. Atom. Nucl. 60 (1997) 2069 [hep-ph/9704301] [INSPIRE].

[45] Yu.A. Simonov, Resolution of the pion puzzle: The QCD string in Nambu-Goldstone mesons, Phys. Atom. Nucl. 67 (2004) 846 [hep-ph/0302090] [INSPIRE].

[46] Yu.A. Simonov, New spectral representation and evaluation of $f(\pi)$ and the quark condensate $<\bar{q} q>$ in the terms of string tension, Phys. Atom. Nucl. 67 (2004) 1027 [hep-ph/0305281] [INSPIRE]. 
[47] S.M. Fedorov and Yu.A Simonov, Pseudoscalar mesons and their radial excitations from the effective chiral Lagrangian, JETP Lett. 78 (2003) 57 [hep-ph/0306216] [INSPIRE].

[48] Yu.A. Simonov, Chiral lagrangian with confinement from the QCD lagrangian, Phys. Rev. D 65 (2002) 094018 [hep-ph/0201170] [INSPIRE].

[49] Yu.A. Simonov, Analysis of the QCD spectrum and chiral symmetry breaking with varying quark masses, Phys. Atom. Nucl. 76 (2013) 525 [arXiv: 1205.0692] [INSPIRE].

[50] M.R. Pennington, Translating quark dynamics into hadron physics (and back again), hep-ph/0207220 [INSPIRE].

[51] T.B. Blum et al., Quenched lattice QCD with domain wall fermions and the chiral limit, Phys. Rev. D 69 (2004) 074502 [hep-lat/0007038] [INSPIRE].

[52] V. Gimenez et al., Operator product expansion and quark condensate from lattice $Q C D$ in coordinate space, Eur. Phys. J. C 41 (2005) 535 [hep-lat/0503001] [INSPIRE].

[53] C. McNeile, An estimate of the chiral condensate from unquenched lattice QCD, Phys. Lett. B 619 (2005) 124 [hep-lat/0504006] [INSPIRE].

[54] G. Colangelo, Finite volume effects in chiral perturbation theory, Nucl. Phys. Proc. Suppl. 140 (2005) 120 [hep-lat/0409111] [InSPIRE].

[55] Yu.A. Simonov and V.I. Shevchenko, Confinement mechanism in the field correlator method, Adv. High Energy Phys. 2009 (2009) 873051 [arXiv:0902 .1405] [INSPIRE].

[56] Yu.A. Simonov, Gluelumps and confinement in QCD, arXiv:1003.3608 [INSPIRE].

[57] Yu.A. Simonov, Analytic calculation of field-strength correlators, Phys. Atom. Nucl. 69 (2006) 528 [hep-ph/0501182] [INSPIRE].

[58] Yu.A. Simonov, Gluelump spectrum in the QCD string model, Nucl. Phys. B 592 (2001) 350 [hep-ph/0003114] [INSPIRE]. 